\title{
Occurrence of Dengue Cases in Silvassa, Dadra Nagar Haveli (Union Territory), India
}

Vikram Khan ${ }^{1}$, Daolatsinh Zala ${ }^{1}$, Sandeep Sanghvi ${ }^{2}$, H.C. Srivastava ${ }^{2}$ and V K. Das ${ }^{1^{*}}$

${ }^{1}$ Directorate of Medical and Health Services, Silvassa-396230, UT of Dadra and Nagar Haveli, India

${ }^{2}$ National Malaria Research Centre (ICMR), Field Station, Nadiad 387001, India

*Corresponding author: VK Das, Directorate of Medical and Health Services, Silvassa-396230, UT of Dadra and Nagar Haveli, India, Tel: +91260-2640615; E-mail: vkdas511@gmail.com

Received date: October 18, 2016; Accepted date: October 24, 2016; Published date: October 28, 2016

Copyright: ( 2016 Das VK, et al. This is an open-access article distributed under the terms of the Creative Commons Attribution License, which permits unrestricted use, distribution, and reproduction in any medium, provided the original author and source are credited.

\begin{abstract}
During last three years increase in dengue incidence was reported from Silvasssa city and adjoining area. We analyzed incidence to determine the occurrence of cases, investigate its causes, and recommend preventative measures for the control. Blood samples were collected from 1583 patients experiencing febrile illness clinically consistent with dengue infection. Serological confirmation of Dengue Infection was done using Dengue ELISA test which detected dengue-specific NS1 antigen, IgM antibody and IgG antibody. Out of 1583 suspected cases, 186 cases $(11.75 \%)$ were confirmed as serologically positive. Contribution of factors was also noted, according to norms. The difference between the proportions of serologically positive cases during different months was significant $(p<$ 0.05). A larger percentage (84.9\%) of serologically positive cases was observed among adults. We also analyzed the association of local and climatic factor with dengue incidence in Silvassa and adjoining area during the year 2012. This retrospective study highlighted rainfall as the major and important climatic factor, which could alone or collectively be associated with infection of Dengue. Vector control strategy must be planned and implemented before onset of monsoon using effective larvicide, involvement of the communities, municipal corporations, industry management and other private sectors.
\end{abstract}

Keywords: Dengue; Dengue hemorrhagic fever; Serology; Ecoepidemiology

\section{Introduction}

Dengue fever (DF) caused by flavi-virus is an important health problem worldwide. Among four distinct serotypes (DEN 1-4), Asian genotypes of DEN-2 and DEN-3 are frequently associated with severe disease accompanying secondary infection. In the last 5 decades, it has invaded many new geographic areas with a 30 fold increase in incidence worldwide WHO, 2009. The countries of the WHO South Asia region and Western Pacific region bear approximately $75 \%$ of the current global disease burden due to Dengue. Since 2003, Dengue Fever (DF) and Dengue Hemorrhagic Fever (DHF) are emerging as major public health problems in India and are reported from more than 19 states. Aedes aegypti and Aedes albopictus are the principal vectors of dengue and are widely prevalent in India. In the last five years, dengue cases have increased 10 folds and death cases have increased 3.5 folds with outbreak/epidemics in many places. This has been known to be endemic in India for over two centuries. In the recent past, high prevalence of DF/DHF and outbreaks had been reported from different states in India [1-4].

There has been a significant increase in dengue morbidity in this Union Territory during the last two years. Since human behavior and change in environment plays important role in the transmission dynamics of mosquito borne disease, this retrospective study was undertaken to determine the dengue incidence in urban and industrial areas and study the association of dengue infection with climatic factors in this geographical region of India.

\section{Material and Methods}

\section{Study area}

Silvassa, the capital city of Dadra Nagar Haveli (Union Territory) is in the western part of India and large scale development, urbanization and industrialization have come up during recent past. Silvassa, the capital city of Dadra Nagar Haveli situated at latitude $-20^{\circ} 54^{\prime} 41^{\prime \prime} \mathrm{N}$ to $20^{\circ} 21^{\prime} 36^{\prime \prime} \mathrm{N}$, Longitude $-72^{\circ} 54^{\prime} 41^{\prime \prime} \mathrm{N}$ to $73^{\circ} 13^{\prime} 13^{\prime \prime} \mathrm{N}$ in the Western Ghat. The $487 \mathrm{sq} \mathrm{km}$ area is hilly, forested, occupied by mainly tribes (population 3.42 lakh) in 72 villages and one town. Owing to subsidiary in taxes, many large (20), medium (564) and small (2118) industries has been established in this Union Territory (UT) of India [2]. Approximately 2.5 lakh skilled and unskilled workforce comes to Silvasssa from dengue and malaria endemic areas of different states of India. The health services are provided to the people by the seven Primary Health Centers (PHC), one Community Health Center (CHC) in rural area and one Civil Hospital at Silvassa with three hundred beds indoor facilities [5-8]. In addition, there are ten private hospitals and fifty medical practitioners. The vector borne disease control programme in the UT is governed and supported by the National Vector Borne Disease Control Programme, New-Delhi.

\section{Patients and diagnosis}

All febrile patients or with a history of fever reported at the outdoor of the department of medicine and emergency wards of Shri Vinoba Bhave Civil Hospital, Silvassa (U.T.) in 2012 were screened for malaria infection. All malaria negative patients who had fever history of 7 days were subjected to diagnosis of dengue with detection of IgM and IgG by ELISA antibody using PanBio (Australia) kits and NS-1 antigen 
ELISA. All6 dengue positive patients were admitted and treated as per the standard guideline (WHO 2009) under supervision of physicians. The clinical history and examination was done by a physician [9-12]. The detailed analysis of 186 dengue cases reported in 2012 was performed. Meteorological data (maximum and minimum temperature and rainfall were obtained) were recorded in the hospital in Silvassa [13].

\section{Entomological investigation}

Monthly entomological surveillance was performed in four sentinel sites (Dadra, Masat, Rakholi and Piparia) and four random sites (Urban, semi-urban/slum and industrial) during the period from January to December 2012 [8,9]. All kinds of breeding habitats in the study area (e.g. Cemented tubs/tanks, overhead tanks, plastic containers, iron/metal drums, junk materials, desert coolers, discarded tyres, coconut shells and curing tanks, etc.) were screened with the help of flash-light and pipette, while bigger containers were searched with the help of a dipper of $300 \mathrm{ml}$ capacity (having white background for better visibility). The type of breeding habitats and their locations were noted. The data on larval survey were analyzed and calculated in terms of different indices like container index (CI), house index (HI) and breteau index (BI) as per the (WHO 1995) procedure. Adult mosquitoes have been sampled with an aspirator and flash torch. Aedes identified up to the species level with the help of standard identification keys [14-16] .

\section{Statistical analysis}

Data were entered and analyzed using Excel 2007 and independently analyzed in SPSS to cross-check for accuracy. Age was recorded in the categorical variable (up to $0-5,6-10,11-15,16-45$, 46-50 and above $>50$ years of age). Data analysis was done using SPSS 17 for Windows. The Chi-square test and correlation coefficient us to compare observed and expected frequencies and association between data $[17,18]$.

\section{Result}

During the last three years (2010 to 2012), annual attendance of outdoor patients in this Civil Hospital has increased from 3.98 lakh to 5.7 lakh. A total of 334, 668 and 1583 suspected DF patients were subjected to sero-diagnosis in 2010, 2011 and 2012 respectively. The sero-posivity during corresponding period ranged between 8.08 to $12.12 \%$.

In 2012, altogether 186 dengue cases were diagnosed at this hospital. Of which 181 (97.31\%) were diagnosed by NS-1, 17 patients were found positive with NS- 1 and IgM and 5 were found positive for IgM by ELISA only. None of them were positive for IgG.

In 2012, dengue had affected all socioeconomic levels of the society and all age groups of the community. The most affected age-group was 16-45 years of age, which was mainly working group of the community and their mobility is normally greater than other age groups $84.9 \%$ (158/186) followed by the children of $\leq 1$ to 15 years of age group $(10.75 \% ; 20 / 186)$. Interestingly, the dengue infection was lowest among the people of more than 45 years of age-group $(4.3 \% ; 8 / 186)$. The variation in the infectivity rate of dengue in different age group is encountered significant $(p \leq 0.05)$. Out of the total dengue cases diagnosed in the hospital, $32.2 \%$ were in migrants working in various firms in this area. These migrants were from the six states of India namely Maharashtra (16.6\%), West Bengal (6.6\%), Gujarat (71.6\%) and 1.66\% each from Karnataka, Uttar Pradesh and Orissa.

The seasonal occurrence of dengue cases in 2011 and 2012 is shown in the Figure 1. The maximum cases were recorded in October to November each year, thereafter sporadic cases were reported till February, indicating that active transmission starts with the onset of monsoon and continues with low intensity of transmission till January. Out of total 186 dengue cases in 2012, 71\% (133) cases where during the monsoon and the remaining cases were in post-monsoon period. The cases among male and female were 142 (76.3\%) and 44 (23.65\%). Significant deviation was reported on sex-wise distribution of dengue cases $(\mathrm{p} \leq 0.05)$.

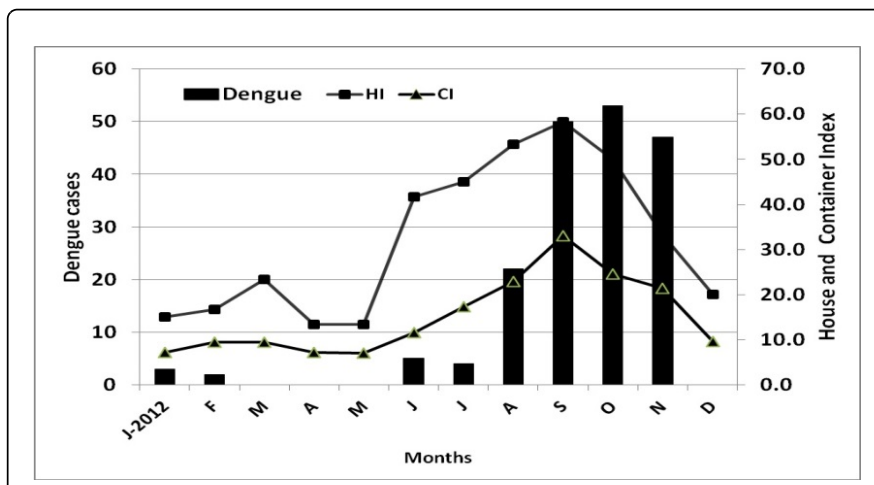

Figure 1: Mosquito breeding indices and dengue cases in Silvassa, (UT).

It was also observed that $36.02 \%(67 / 186)$ of the total dengue patients were residing in the factories or adjoining campus. The Amli (33), Masat (28) and Rakholi (26) factories had highest number of dengue cases in 2012. The mean age of these patients was 23 years.

The 60 houses each in sentinel and random sites were examined for Aedes breeding in different type of temporary and permanent water bodies, both indoors and outdoors in residential and commercial areas of the town. Aedes aegypti was found the dominant species in Silvassa, The mean adult Aedes density were 8.28 per man/ hour (range from 2.7 to 16.2). There appear to be clear, significant correlation between the adult Aedes density and the incidence of dengue $(r=0.65 ; \mathrm{p} \leq 0.05)$.

Further analysis also showed a significant relationship between larval index (Breteau Index, Container Index and House Index) and the dengue incidence during 2012 (CI: $r=0.89, p \leq 0.05 ; \mathrm{HI}: \mathrm{r}=0.70, \mathrm{p} \leq$ 0.05 ; and BI: $r=0.70, p=0.05$ ). The monthly average of HI, CI, BI and adult mosquito density are presented in Figure 1 . Both the adult density and the breeding of Aedes were observed in these localities throughout the year, the peak per man per hour density (13.3 MHD) appeared in (June to October) ranging 10.2-16.2 MHD. Similarly, the highest HI (58.3), CI (33.1), and BI (216) were also recorded in September and were low during December to May. The Container Index in Silvassa had been reported above the critical level during the monsoon period and the post monsoon period in town as well as in the campuses of factories and nearby human habitats. Aedes aegypti also breeds in artificial and discarded containers in this area. 


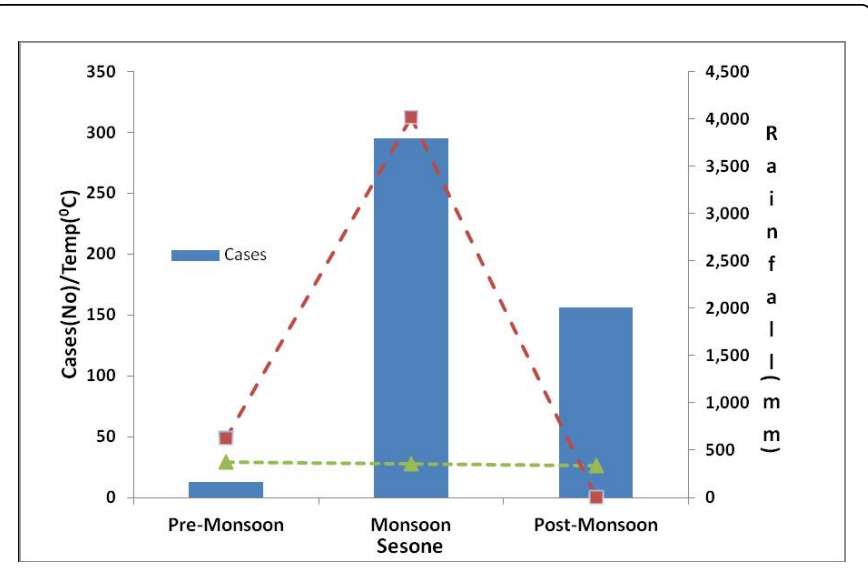

Figure 2: Seasonal prevalence of dengue, temperature and rainfall in Silvassa during 2012-2013.

The annual average outdoor maximum and minimum air temperature in 2012 was $32.10^{\circ} \mathrm{C}\left(29.77^{\circ} \mathrm{C}-33.73^{\circ} \mathrm{C}\right)$ and $22.04^{\circ} \mathrm{C}$ $\left(15.06^{\circ} \mathrm{C}-26.42^{\circ} \mathrm{C}\right)$ respectively. During pre-monsoon (March to June), maximum and minimum outdoor air temperature ranged between $32.2 \mathrm{C}$ to $33.7 \mathrm{C}$ and $18.9^{\circ} \mathrm{C}$ to $26.4^{\circ} \mathrm{C}$ respectively. In monsoon and post monsoon period, maximum and minimum temperature ranged $30.4^{\circ} \mathrm{C}$ to $33.6^{\circ} \mathrm{C}$ and $23.6^{\circ} \mathrm{C}$ to $25.7^{\circ} \mathrm{C}$ and $29.7^{\circ} \mathrm{C}$ to $33.1^{\circ} \mathrm{C}$ and $15.0^{\circ} \mathrm{C}$ to $19.2^{\circ} \mathrm{C}$ respectively. The correlation between dengue cases and temperature ( $\mathrm{r}=-0.11$ and 0.05 probability) was insignificant (Figure 2). During last three years (2010-2012), Silvassa received highest rainfall $(2745.4 \mathrm{~mm})$ in 2011 while lowest of $1598.8 \mathrm{~mm}$ in 2012 . It started in June and variable degree of rainfall was recorded till October makes prolonged favorable macro as well as micro climatic conditions for the proliferation of the dengue vector, Aedes aegypti and many potential vector breeding sites are created in the town, factories campus and adjoining areas.

Dengue cases were highest (69.4\%) during the monsoon followed by post-monsoon $(28.0 \%)$ when the mean minimum temperature was higher $\left(24.9^{\circ} \mathrm{C}\right)$ as compared to post-monsoon $\left(17.2^{\circ} \mathrm{C}\right)$ and mean maximum temperature was nearly at par. But the precipitation was highest during the monsoon period. The analysis also showed insignificant correlation between rainfall and dengue cases $(r=-0.02$; $\mathrm{p}=0.05)$. The dengue incidences started occurring with the onset of rainfall and reached to highest level in post monsoon.

\section{Discussion}

Association of environmental conditions with dengue incidence had been reported by scholars in different region. The extrinsic incubation period of dengue is also influenced in part by climatic conditions, effecting vector survivorship and viral transmission. Our retrospective analysis of dengue incidence and certain environmental factors, especially rainfall and temperature in this geographic area has provided understanding of disease pattern in the community and its association with climatic factors. In Silvassa, the number of dengue cases reported annually by civil hospital in 2010-2012 increased sharply but the seropositive rate remained nearly similar. The data used in the present study were derived from the public hospital, cases diagnosed and treated at the private clinic/hospital were not included in the analysis, hence further study is required to know the actual disease burden of the Silvassa. In general, all age groups of the community suffered with dengue infection. The dengue infection in children $(<15)$ age group was low in contrast to reports from other states. The most suffered group of the community was 16-45 years of age group. The people of this age-group are earning member/student/ workers in the family. They work in factories and other places where contact/or exposure to mosquito bite is greater. The higher seropositivity among males was found statistically significant $(\mathrm{p}<0.001)$. Indian traditional dressing among female might have provided more protection against contact with vector Aedes aegypti that bite mainly during day time. Aedes aegypti breeds in artificial or natural water storage containers in near vicinity of the human habitats in this area. Similar, the breeding habit of this vector had also been reported by other authors. The localities with higher population have more opportunities for its breeding, vector abundance and feeding on human. Therefore, Silvassa town had more dengue incidence as compared to the factory or nearby campuses.

In general, dengue cases were reported throughout the year in varying proportion. But the majority of the cases was reported in monsoon and the post monsoon season. The results also showed that minimum temperature and rainfall have no direct influence on dengue incidence in monsoon. The prolonged rainfall and ambient temperature had resulted in large numbers of mosquito breeding sites, especially outdoors, and longer survivorship of the Aedes aegypti. The dengue cases diagnosed by NS-1 were in late monsoon and post monsoon period indicated active transmission of dengue virus. The occurrence of dengue had no strong correlation with temperature and rainfall, but large numbers of breeding sites in monsoon and post monsoon period along with factors like industrialization and sociological aspects has influenced the dengue transmission in this area.

\section{Conclusions}

In conclusion, the study provides insight of the dengue occurrence in Silvassa. It will help in preparing and formulating control strategies using effective vector control methods in urban and industrial area. There is a strong need to block the transmission to prevent morbidity. The present dengue control strategy employed by public health department is reactive through case management and vector control by using fogging and larvicides. A part of implementation of integrated vector management strategy before onset of monsoon, involvement of the communities, municipal corporations, industry management and other private sectors should be important for dengue control in this area. In this hilly and forested terrain, further study of the ecological, sociological and biological factors involved in dengue transmission would help in making the most effective strategies for dengue control.

\section{Acknowledgments}

We thank to the Mission Director, NRHM, the UT of Dadra and Nagar Haveli, Silvassa and Integrated Disease Surveillance Programme which provided the laboratory facilities and other necessary support. This paper bears the National Institute of Malaria Research, New Delhi, India publication screening committee approval no. 035/2013.

\section{References}

1. Baig HZ, Nawaz AJ (2012) Climatic factors affecting dengue fever incidence in Lahore, Pakistan. WHO Dengue Bull 36: 64-72. 
Citation: Das VK, Zala DS, Sanghvi S, Srivastava HC (2016) Occurrence of Dengue Cases in Silvassa, Dadra Nagar Haveli (Union Territory), India. J Trop Dis 4: 221. doi:10.4172/2329-891X.1000221

Page 4 of 4

2. Balakrishnan N, Venkatesh S, Lal S (2006) An entomological study on the dengue vectors during outbreak of dengue in Triuppur town and its surrounding, Tamil Nadu, India. J Commun Dis 38: 164-168.

3. Bhatt S, Peter WG, Oliver JB, Jane PM, Andrew WF, et al. (2013) The global distribution and burden of dengue.

4. Das BP, Kaul SM (1998) Pictorial key to the common Indian species of Aedes (Stegomyia) mosquitoes. J Commun Dis 30: 123-127.

5. Focks DA (2003) A Review of Entomological Sampling Methods and Indicators for Dengue Vectors. Gainesville.World Health Org.

6. Foo LC, Lim TW, Lee HL, Fang R (1985) Rainfall, abundance of Aedes and dengue infection in Selangor, Malaysia. J Trop Med Pub Health 16: 560-588.

7. Fuller DO, Troyo A, Beier JC (2009) El Nino southern oscillation and vegetation dynamics as predictors of dengue fever cases in Costa Rica. Environ Res Lett.

8. Gunasekaran K, Kaveri SM, Kavita A, Suresh BBV, Padma PP, et al. (2011) Dengue disease status in Chennai : A retrospective analysis. Indian J Med Res 133: 322-325.

9. Hati AK (2009) Dengue serosurveillance in Kolkata, facing an epidemic in West Bengal. India J Vector Borne Dis 46: 197-204.

10. Johannson MA, Dominici F, Glass GE (2009) Local and global effects of climate on dengue transmission in Puerto Rico. PLoS Neg Trop Dis 3: 382 .
11. Kader AMS, Fernando J, Kiruba J, Palani G, Sarangapani GTD, et al. (1998) Investigation of Aedes ageypti breeding during dengue fever outbreak in villages of Dharmpuri district, Tamil Nadu, India. Dengue Bull 22: 40-45.

12. Khan V, Zala DB, Srivastava HC (2013) Entomological indicators during transmission season of dengue in Silvassa (India). J Parasit Dis.

13. Kumar A, Sharma SK, Padbidri VS,Takare JP, Jain DC, et al. (2001) An outbreak of dengue fever in rural areas of northern India. J Commun Dis 33: 274-281.

14. Mahadev PVM, Kollali VV, Rawal ML, Pujara PK, Shikh BH, et al. (1993) Dengue in Gujarat State, India. J Med Res 97: 135-144.

15. Tarphdar D, Sarkar A, Mukhopadhyay BB, Chatterjee S (2012) Short Report: A comparative study of clinical features between monotypic and dual infection cases with chikungunya virus and dengue virus in West Bengal, India. Am J Trop Med Hyg 86: 720-723.

16. Vijayakumar TS, Chandy S, Sathish N, Abraham M, Abraham P, et al. (2005) Is dengue emerging as a major public health problem?. Indian J Med Res 12: 100-107.

17. World Health Organization (1995) Guidelines for dengue surveillance and mosquito control. Western Pacific Education in Action series No.8.

18. World Health Organization (2009) Dengue Guidelines for diagnosis, treatment, prevention and control. 